\title{
Praxisführung für Grundversorger/innen
}

Bisher findet keine gezielte «Managementweiterbildung» der zukünftigen Hausärztinnen/-ärzte statt. Es gibt zwar vielfältige kommerzielle Angebote, in denen sich ein Assistenzarzt Informationen und Hilfe zur Praxiseröffnung und Praxisführung holen kann, viele wichtige Aspekte zur Führung einer eigenen Praxis werden dabei jedoch nicht berücksichtigt. Hier versucht nun das KHM neben dem Angebot «Praxisassistenz» mit einem neuen Angebot die Lücke zu schliessen. Nach intensiven Vorarbeiten konnte 2004/2005 erstmals ein Pilotkurs mit initial 21 Teilnehmerinnen und Teilnehmern mit Erfolg durchgeführt werden. Gestützt auf die Evaluation dieses Kurses, wird im laufenden Jahr das Kursmodul in optimierter Form erneut angeboten.

Das Weiterbildungsmodul Praxisführung ist berufsbegleitend über eine Dauer von 6 Monaten angelegt und versucht moderne Grundsätze der Erwachsenenbildung zu berücksichtigen. Jeder Teilnehmer analysiert vor dem Kursbeginn seine individuellen Bedürfnisse und Lücken mit Hilfe eines speziell für die hausärztliche Praxisführung entwickelten Instruments. Daraus ergibt sich ein individueller Lernplan, der in den Präsenzteilen und in der Selbstlernphase umgesetzt wird. Dieses «self-assessment» umfasst wichtige Fragen und Stichworte $\mathrm{zu}$ folgenden Themen:

- Selbstmanagement: Hier geht es um Zeitund Aufgabengestaltung, Umgang mit persönlichen und beruflichen Belastungen, Stressmanagement und Burn-out-Prophylaxe, Gleichgewicht zwischen Beruf und anderen Lebensbereichen, neue Arbeitszeit- und Praxismodelle, Massnahmen zur Erhaltung und Förderung der beruflichen Kompetenz.

- Der Bereich Praxismanagement umfasst die ganze Breite der unternehmerischen Aufgaben: Menschenführung (Personalselektion, -führung und -entwicklung; Teamarbeit; Kommunikation und Umgang mit Patienten); Demand-, Disease- und Case-Management; die Organisation des Praxisbetriebes (Betrieb, Finanzen, Informationsfluss, Dokumentation).
- Die Vernetzung zwischen Praxis und Umfeld wird immer wichtiger: Zusammenarbeit mit Kollegen, medizinischen und sozialen Diensten, Behörden, Versicherungen; Aufgaben für die öffentliche Gesundheit; berufs- und allgemeinpolitisches Engagement inkl. dafür erforderlicher Kommunikationsfertigkeiten, Ethik, Qualitätssicherung und Praxisforschung, Umweltanliegen.

Im ersten Präsenzkurs werden die Grundlagen für eine erfolgreiche Selbst- und Gruppenlernphase gelegt und einige wesentliche Kerninhalte im Bereich Selbstmanagement und zu Praxisformen (Einzelpraxis, Gruppenpraxis) vermittelt (Dauer anderthalb Tage).

In der Selbst- und Gruppenlernphase arbeiten die Teilnehmenden, unterstützt durch die Kursleitung, über eine Dauer von 5 Monaten, um ihre individuellen Lernziele zu erreichen. Sie lernen selbständig und tauschen Informationen über E-Mail und über die Webplattform des Weiterbildungsmoduls aus. Literatur und Erfahrungsberichte sowie eigene Arbeiten können über die webbasierte Dokumentationsplattform allen Teilnehmenden zur Verfügung gestellt werden. Die Webplattform enthält auch weitere Kursunterlagen, Begleitmaterialien und Literatur, Links, Adresslisten und eine Datenbank mit Drittangeboten. Auf Wunsch besteht die Möglichkeit von Webmeetings, und die Kursleitung unterstützt auch Gruppentreffen.

Im zweiten Präsenzkurs von wiederum anderthalb Tagen präsentieren die Teilnehmerinnen und Teilnehmer ihre Erfahrungen und Arbeiten den anderen Kolleginnen und Kollegen des Kurses. Es sollen eine intensive Diskussion und ein intensiver Erfahrungsaustausch stattfinden. Bei Bedarf können externe Experten zugezogen werden.

Die Projektgruppe wird parallel zu den Kursen eine Wissensdatenbank und eine Linksammlung zu Fragen der Praxisführung aufbauen. Es bestehen auch Überlegungen, das Kursmodell später auf andere Inhalte zu übertragen, mit dem 
Ziel, eine bessere Strukturierung der Weiterbildung zum Hausarzt zu ermöglichen. Schliesslich ist zu einem späteren Zeitpunkt auch ein Kurs für bereits etablierte Hausärzte in der gleichen Form zum Thema «Praxisführung» denkbar.

\section{Teilnahmebedingungen}

In Weiterbildung zur Hausärztin / zum Hausarzt (Allgemeinmedizin, Innere Medizin oder Pädiatrie) etwa 1-3 Jahre vor der Praxiseröffnung. Zugang zum Internet und gute E-Mail-Kommunikation. Kurssprache: Deutsch.

\section{Kursgebühr}

Fr. 800.-. Die Kosten für die Unterkunft und Verpflegung (etwa Fr. 200.-) an den Präsenzkursen sind durch die Teilnehmer direkt vor Ort zu bezahlen.

\section{Termine}

Anmeldefrist bis Ende April 2006.

- Präsenzteil 1: 25./26.8.2006, Kappel a.A.;

- Präsenzteil 2: 19./20.1.2007, Kappel a.A.

\section{Informationen und Anmeldung}

Kollegium für Hausarztmedizin, Landhausweg 26, 3007 Bern, www.kollegium.ch/pdf (Infoflyer und Anmeldetalon zum Herunterladen). 